\title{
Ardisia Crenata Sims Var. Bicolor Physiological Attributes And Soil Physico-Chemical Properties Along An Altitudinal Gradient On The Gaoligong Mountain, Southwest China
}

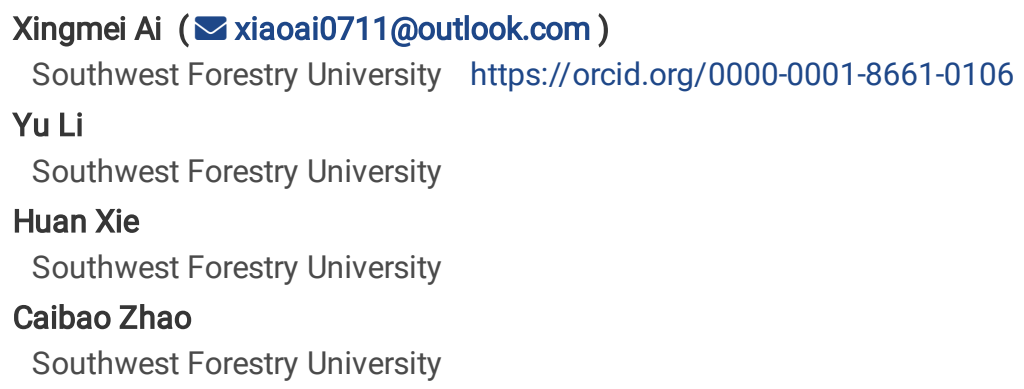




\section{Abstract}

Aims This research investigated the acclimation of Ardisia crenata Sims var. bicolor at different elevations, and evaluated both soil and plant responses to altitude.

Methods We collected rhizosphere soil and upper canopy leaves from this evergreen shrub during the plant blooming phase from six different altitudinal ranges on the Gaoligong Mountain. We measured soil nutrients and enzyme activities. Spectrophotometry was used to investigate twelve indicators of leaves, including the antioxidant enzyme activities.

Results Soil physicochemical analysis showed that soil pH was negatively correlated with altitude and decreased with soil depth, whereas there were positive correlations between altitude and soil water content, organic matter, and soil nutrients, including organic carbon, total $\mathrm{N}$, and total P. Total K at high elevations showed an increase with soil depth but not significant. Soil urease, saccharase, amylase, catalase, and peroxidase activities were significantly positively correlated with altitude and organic matter, and these enzymes were sensitive to environmental changes. In contrast, specific enzyme activity provided more ecological information and responded more clearly to altitudinal changes, thus playing a crucial role in the study of interactions between soil nutrients and enzymes. Ardisia crenata var. bicolor showed significant differences in physiological compounds (except for anthocyanin); the concentrations of all compounds increased with altitude.

Conclusions Our results indicate that plant-soil interactions with elevation changes significantly affect plant distribution and restrict plant growth and reproduction, which contribute to an understanding of the habitat and physiological characteristics and provide a theoretical basis for the effective cultivation and management of this species.

\section{Introduction}

Ardisia crenata Sims var. bicolor (Primulaceae) is an evergreen understory shrub, found in tropical and subtropical hardwood forests. This shrub produces an abundance of attractive red fruit and exhibits viviparous germination. After shedding, dense breeding areas (up to 300 stems per $\mathrm{m}^{2}$ ) are formed which, due to long-term natural selection in response to lower light penetration in the understory, maintain an efficient population structure and improve the shade tolerance of the seedlings (Kitajima et al. 2006). Ardisia crenata var. bicolor is a highly valued ornamental and medicinal plant. It is widely cultivated as an undergrowth companion species in gardens and as a house plant. Medicinally, its phytochemical constituents possess significant anti-tumor, anti-cancer, and anti-inflammatory properties (Kobayashi and de Mejía 2005; Liu et al. 2016; Ma et al. 2015; Podolak et al. 2019; Raina et al. 2014).

In recent years, wild sources of $A$. crenata var. bicolor have been heavily exploited and natural populations are rapidly declining, potentially altering soil chemical properties and plant growth in these regions (Körner 2007). In general, changes in soil properties along altitudinal gradients are thought to be caused by environmental factors including atmospheric pressure, temperature, rainfall, photoperiod, UV radiation, and oxygen levels (Ahmad et al. 2016; Chen et al. 2014; Chen et al. 2018;

Terfa et al. 2014). In addition, various plants can alter certain physicochemical properties of the soil along altitudinal gradients, depending on different vegetation types as well as root residues (Sundqvist et al. 2014; Ushio et al. 2010). The greater the vegetation abundance and root secretions, the higher the soil organic carbon (SOC), nitrogen, and phosphorus content (Herold et al. 2014), and the greater the activity of soil enzymes involved in the transformation and cycling of nutrients (Hedo et al. 2014). Soil enzymes are sensitive to environmental changes. They act on substances produced by microorganisms or secreted from animal or plant residues in the soil ecosystem (Burns et al. 2013). Soil enzymes respond to soil nutrient levels and can be used as soil fertility indicators (Saggar et al. 1999; Trasar-Cepeda et al. 2008). The specific enzyme activity per unit of SOC (SOCE) is known to provide more ecological information and respond more accurately to altitudinal changes than absolute enzyme activity (Raiesi and Beheshti 2014; Xiao et al. 2018; Zhang et al. 2015).

There is evidence of morphological variation in plants along altitudinal gradients. Sometimes these variations are based predominantly on genetics, but more often they are environmentally based (Byars et al. 2007). Therefore, the changes in environmental factors that occur at different altitudes provide an exploratory way to study the adaptability of plants (De Frenne et al. 2013). To deal with oxidative damage under adverse environmental conditions, plants growing at different altitudes can regularly undergo physiological and metabolic changes through mechanisms such as the antioxidant enzyme defense system and osmotic adjustments that protect cells from oxidative stress through free radicle scavenging (Mittler 2002). These mechanisms help plants tolerate stress and acclimatize effectively (Cui et al. 2016). Vegetation in areas with high biodiversity is directly and indirectly affected by anthropogenic actions and altitude, as occurs in the Gaoligong Mountain region. We studied the physiological characteristics of $A$. crenata var. bicolor in this region as an index for the evaluation of plant adaptive strategies. Given that the Gaoligong Mountain population of A. crenata var. bicolor is randomly distributed and has high adaptability to the understory environment, several physiological indexes and the activity of a number of enzymes were measured to elucidate how this species copes with oxidative stress. We tested the hypothesis that the physiological mechanisms of $A$. crenata var. 
bicolor and soil properties are significantly affected by altitudinal gradients on the Gaoligong Mountain, with a view to determining how such a plant population functions at different altitudes.

\section{Materials And Methods}

Study area

Gaoligong Mountain is characterized by highly variable climatic conditions and a rich biodiversity. The study area was located on the east slope of the Gaoligong Mountain, on the west side of the Nujiang river within Baoshan city $\left(24^{\circ} 56^{\prime} 39^{\prime \prime} \mathrm{N}-24^{\circ} 57^{\prime} 03^{\prime \prime} \mathrm{N}, 98^{\circ} 45^{\prime} 17^{\prime \prime} \mathrm{E}-\right.$

$\left.98^{\circ} 49^{\prime} 19^{\prime \prime E}\right)$, Yunnan province, southwest China (Fig. 1). The elevation of the area ranges from $670 \mathrm{~m}$ a.s.I. in the Nujiang river valley to $3520 \mathrm{~m}$ a.s.I. in the subalpine meadows, and it has a low-latitude plateau monsoon climate with a mean annual temperature of $12.8{ }^{\circ} \mathrm{C}$ and an annual precipitation of 1,369.7 mm (according to 2016 records from the Gaoligong Mountain meteorological station). The diurnal temperature variation is marked, and the area experiences distinct dry and wet seasons. The soil and

vegetation types are vertically distributed along the altitudinal gradient. The soils are red, yellow-red,

yellow-brown, brown, dark-brown, and subalpine shrub meadow soils, while the vegetation types

mainly include tropical monsoon rainforest, monsoon evergreen broad-leaved forest (<1,700 m a.s.l.), humid evergreen broad-leaved forest (1,800-2,600 $\mathrm{m}$ a.s.I.), coniferous forest (2,700-3,100 $\mathrm{m}$ a.s.I.), and alpine shrub vegetation and meadow (>3,200 $\mathrm{m}$ a.s.I.).

Soil sampling

To evaluate the effects of elevation on soil properties and enzyme activity, $A$. crenata var. bicolor rhizosphere soil was sampled at six sites along an altitudinal gradient, from low altitude tropical monsoon rainforest to the mid-mountain humid evergreen broad-leaved forest.

Sampling sites were located at elevations of 1,257 m, 1,538 m, 1,744 m, 1,970 m, 2,135 m, and 2,376 m a.s.l. and a geographical positioning system was used to record altitude and aspect. Detailed descriptions of the sites are shown in Table 1 . Three replicate $15 \times 15 \mathrm{~m}$ plots were selected at each site. For determination of soil physicochemical properties, undisturbed $A$. crenata var. bicolor rhizosphere soil in each plot was collected from depths of $0-20 \mathrm{~cm}$ and $20-40 \mathrm{~cm}$, using the S-type distribution method with a standard cutting ring. A total of five $2-\mathrm{kg}$ soil cores were collected from each plot and mixed into one composite soil sample by quartering and the removal of visible roots, rocks, and litter. The samples were stored in polyethylene bags and labeled. For laboratory analysis, each mixed soil sample was divided into two parts: one was kept at $4^{\circ} \mathrm{C}$ for determination of enzyme activity, the other was air-dried, and then passed through a 0.25 -mm sieve in preparation for the analysis of soil physicochemical properties. All laboratory analyses of the prepared soil samples were repeated three times. 
Table 1

Descriptions of the sampling sites

\begin{tabular}{|c|c|c|c|c|c|c|}
\hline Site & Vegetation type & $\begin{array}{l}\text { Altitude } \\
\text { (m) }\end{array}$ & $\begin{array}{l}\text { Longitude } \\
\text { and } \\
\text { latitude }\end{array}$ & $\begin{array}{l}\text { Direction } \\
\text { of slope }\end{array}$ & $\begin{array}{l}\text { Soil } \\
\text { type }\end{array}$ & Dominated vegetation \\
\hline 1 & $\begin{array}{l}\text { Tropical monsoon } \\
\text { rainforest }\end{array}$ & 1257 & $\begin{array}{l}\mathrm{N} 24^{\circ} 56^{\prime} 54^{\prime \prime} \\
\mathrm{E} 98^{\circ} 49^{\prime} 19^{\prime \prime}\end{array}$ & East & $\begin{array}{l}\text { brown } \\
\text { red }\end{array}$ & $\begin{array}{l}\text { Machilus yunnanensis, Rhododendron delavayi, } \\
\text { Viburnum oliganthum, Quercus acutissima, Phyllanthus } \\
\text { emblica, Nephrolepis cordifolia, Rhododendron delavayi }\end{array}$ \\
\hline 2 & $\begin{array}{l}\text { Monsoon } \\
\text { evergreen broad- } \\
\text { leaved forest }\end{array}$ & 1538 & $\begin{array}{l}\mathrm{N} 24^{\circ} 56^{\prime} 60^{\prime \prime} \\
\mathrm{E} 98^{\circ} 48^{\prime} 33^{\prime \prime}\end{array}$ & East & $\begin{array}{l}\text { Yellow } \\
\text { red }\end{array}$ & $\begin{array}{l}\text { Cyclobalanopsis glauca, Castanopsis echnocarpa, } \\
\text { Gordonia axillaris, Viburnum oliganthum }\end{array}$ \\
\hline 3 & $\begin{array}{l}\text { Semi-humid } \\
\text { evergreen broad- } \\
\text { leaved forest }\end{array}$ & 1744 & $\begin{array}{l}\mathrm{N} 24^{\circ} 56^{\prime} 39^{\prime \prime} \\
\mathrm{E} 98^{\circ} 46^{\prime} 51^{\prime \prime}\end{array}$ & Southeast & $\begin{array}{l}\text { Yellow } \\
\text { red }\end{array}$ & $\begin{array}{l}\text { Cyclobalanopsis glauca, Castanopsis delavayi, } \\
\text { Castanopsis concolor, Quercus acutissima, Lithocarpus } \\
\text { hancei, Schima superba }\end{array}$ \\
\hline 4 & $\begin{array}{l}\text { Semi-humid } \\
\text { evergreen broad- } \\
\text { leaved forest }\end{array}$ & 1970 & $\begin{array}{l}\mathrm{N} 24^{\circ} 56^{\prime} 47^{\prime \prime} \\
\mathrm{E} 98^{\circ} 46^{\prime} 27^{\prime \prime}\end{array}$ & Southeast & $\begin{array}{l}\text { Yellow } \\
\text { red }\end{array}$ & $\begin{array}{l}\text { Cyclobalanopsis glauca, Castanopsis concolor, Quercus } \\
\text { acutissima }\end{array}$ \\
\hline 5 & $\begin{array}{l}\text { Mid-mountain } \\
\text { humid evergreen } \\
\text { broad-leaved forest }\end{array}$ & 2135 & $\begin{array}{l}\mathrm{N} 24^{\circ} 56^{\prime} 46^{\prime \prime} \\
\mathrm{E} 98^{\circ} 46^{\prime} 04^{\prime \prime}\end{array}$ & East & $\begin{array}{l}\text { Yellow } \\
\text { brown }\end{array}$ & $\begin{array}{l}\text { Cyclobalanopsis glauca, Lithocarpus glaber, } \\
\text { Rhododendron protistum, Sabina pingii, Fargesia } \\
\text { spathacea, Ternstroemia gymnanthera, Elaeagnus } \\
\text { pungens, Viburnum oliganthum, Betula forest, } \\
\text { Castanopsis concolo, Castanopsis orthacantha }\end{array}$ \\
\hline 6 & $\begin{array}{l}\text { Mid-mountain } \\
\text { humid evergreen } \\
\text { broad-leaved forest }\end{array}$ & 2376 & $\begin{array}{l}\mathrm{N} 24^{\circ} 57^{\prime} 03^{\prime \prime} \\
\mathrm{E} 98^{\circ} 45^{\prime} 17^{\prime \prime}\end{array}$ & Southeast & $\begin{array}{l}\text { Yellow } \\
\text { brown }\end{array}$ & $\begin{array}{l}\text { Lithocarpus hancei, Alcimandra cathcartii, Manglietia } \\
\text { insignis, Cyclobalanopsis glauca, Castanopsis hystrix, } \\
\text { Litsea cuosma, Gordonia longicarpa, Aidia shweliensis }\end{array}$ \\
\hline
\end{tabular}

Laboratory analyses

The soil physicochemical analyses were performed following the methods described in Chodak et al. (2015), including for soil organic matter (OM). Soil water content (SWC) was calculated based on the weight of soil samples before and after oven-drying (at $105 \pm 2{ }^{\circ} \mathrm{C}$ ) for at least $72 \mathrm{~h}$. Soil pH was measured using a potentiometric method in a 2.5:1 (w/v) ratio of substrate to distilled water suspension (De Feudis et al. 2016). The SOC content was determined using $\mathrm{H}_{2} \mathrm{SO}_{4}-\mathrm{K}_{2} \mathrm{Cr}_{2} \mathrm{O}_{7}$ oxidation (Bao 2008). Total nitrogen (TN) was determined by the Kjeldahl method (Brzezińska et al. 2011). Total phosphorus (TP) was determined calorimetrically after wet digestion with $\mathrm{H}_{2} \mathrm{SO}_{4}-\mathrm{HClO}_{4}$, and total potassium (TK) was determined with a flame photometer (Bao 2008).

Soil enzyme activity assays were based on colorimetric determination of the products released by the enzymes. Soil urease (URE) activity was measured by indophenol colorimetry with urea as the substrate, saccharase (SAC) activity was measured using the 3, 5-dinitrosalicylic acid colorimetry method, and amylase (AMY) by anthrone colorimetry. Catalase (CAT) activity was measured using $0.3 \%$ hydrogen peroxide solution as a substrate; the residual $\mathrm{H}_{2} \mathrm{O}_{2}$ was determined by titration with $0.02 \mathrm{M} \mathrm{KMnO}_{4}$ under acidic conditions. Peroxidase (POD) activity was determined by pyrogallol colorimetry (Guan 1986; Ren et al. 2016). The SOCE was calculated by dividing enzyme activity by SOC content to normalize the activity (Trasar-Cepeda et al. 2008; Zhang et al. 2015).

Plant sample collection and physiological attributes

Leaf samples were collected at the same six elevations as the soil samples. Expanded and intact fresh upper leaves of similar size and age were collected in the blooming season (May 2019). Leaf sampling was replicated three times at each sampling site. The sampled leaves were wrapped in aluminum foil and immediately immersed in liquid nitrogen. Subsequently, they were stored at $-80^{\circ} \mathrm{C}$ pending laboratory analysis. Chlorophyll content was analyzed following Wellburn and Lichtenthaler (1984) after extraction in acetone (80\%, v/v), and the absorbance of chlorophyll a (Chl a), and chlorophyll b (Chl b) was measured at 663 and 645 nm using a UV-721 spectrophotometer. Total chlorophyll (Chl t) concentration was calculated as follows: Chl t $=8.02 \times \mathrm{OD}_{663}+20.20 \times \mathrm{OD}_{645}$. The anthocyanin content (ANT) of the leaves was determined using the method described by Kim et al. (2011). The leaf protein content (PRO) was determined by the Coomassie brilliant blue G-250 method which uses bovine serum albumin as a standard $\left(100 \mu \mathrm{g} \cdot \mathrm{mL}^{-1}\right)$. The soluble sugar (SS) content of the leaves was determined following Li (2000) and the starch content (ST) was measured as the residue from the soluble sugar. To investigate the enzymatic antioxidants superoxide dismutase (SOD), POD, and CAT, they were first extracted using $50 \mathrm{mM}$ phosphate buffer (pH 7.8, $5 \mathrm{~mL}$ ) and centrifuged $(8,000 \times \mathrm{g})$ for $20 \mathrm{~min}$. The activity of the supernatant enzymes was determined following Li (2000). Polyphenol oxidase 
(PPO) activity was measured by catechol oxidation, and phenylalanine ammonia-lyase (PAL) activity was determined following McCallum and Walker (1990).

Statistical analyses

Statistical analyses were performed using Microsoft Excel and SPSS software (Version 21.0). The soil physicochemical properties, enzyme activity, and plant physiological responses were compared with elevation by using a one-way ANOVA. A Pearson's correlation was conducted to evaluate the relationships between environmental factors, soil enzyme properties, and plant physiological characteristics. Redundancy analysis (RDA) using CANOCO (v.5.0 for Windows) was performed to determine the associations between soil enzyme activity and SOCE and plant physiological characteristics and soil nutrients. The means and standard deviations of three replicates per variable and Tukey's post-hoc $(P<0.05)$ test were used to identify the values with significant differences.

\section{Results}

Variations in soil physicochemical properties along the altitudinal gradient

When we collected the soil samples, we found that the perennial populations of $A$. crenata var. bicolor at different altitudes showed no significant differences in rooting depth. Roots were mostly distributed from $0-40 \mathrm{~cm}$, indicating that altitudinal variation had little effect on the growth of roots.

However, the soil physicochemical characteristics of mainly the yellow-red and yellow-brown soil types varied significantly with elevation (Table 2). The soil pH was below 6.0 at all elevations (mean pH 5.12) and showed a decrease of 1.48 from low elevation (1,257 $\mathrm{m}$ a.s.I.) to high elevation (2,376 $\mathrm{m}$ a.s.I.). There was a slight increase in $\mathrm{pH}$ with soil depth at all sites. Correspondingly, SWC varied 
Table 2

Soil physicochemical characteristics varied with altitudinal gradients

\begin{tabular}{|c|c|c|c|c|c|c|c|c|c|}
\hline Altitude & $\begin{array}{l}\text { soil } \\
\text { layer }\end{array}$ & $\mathrm{pH}$ & $\begin{array}{l}\text { SWC } \\
\%\end{array}$ & $\begin{array}{l}\text { OM } \\
(\mathrm{g} / \mathrm{kg})\end{array}$ & $\begin{array}{l}\text { TN } \\
(\mathrm{g} / \mathrm{kg})\end{array}$ & $\begin{array}{l}\text { TP } \\
(\mathrm{g} / \mathrm{kg})\end{array}$ & $\begin{array}{l}\text { TK } \\
(\mathrm{g} / \mathrm{kg})\end{array}$ & $\begin{array}{l}\text { SOC } \\
(\mathrm{g} / \mathrm{kg})\end{array}$ & $\mathrm{C} / \mathrm{N}$ \\
\hline \multirow[t]{3}{*}{$1257 m$} & $\begin{array}{l}0 \sim \\
20 \mathrm{~cm}\end{array}$ & $\begin{array}{l}5.94 \pm \\
0.00 \mathrm{aA}\end{array}$ & $\begin{array}{l}22.59 \pm \\
0.91 \mathrm{dA}\end{array}$ & $\begin{array}{l}70.38 \pm \\
0.37 \mathrm{dA}\end{array}$ & $\begin{array}{l}2.15 \pm \\
0.03 \mathrm{~d} A\end{array}$ & $\begin{array}{l}0.56 \pm \\
0.03 \mathrm{dA}\end{array}$ & $\begin{array}{l}10.28 \pm \\
0.28 \mathrm{dA}\end{array}$ & $\begin{array}{l}20.37 \pm \\
0.77 f A\end{array}$ & $\begin{array}{l}9.46 \pm \\
0.38 \mathrm{dA}\end{array}$ \\
\hline & $\begin{array}{l}20 \sim \\
40 \mathrm{~cm}\end{array}$ & $\begin{array}{l}5.98 \pm \\
0.00 \mathrm{aA}\end{array}$ & $\begin{array}{l}24.03 \pm \\
0.90 \mathrm{dA}\end{array}$ & $\begin{array}{l}44.51 \pm \\
0.26 \mathrm{~dB}\end{array}$ & $\begin{array}{l}1.93 \pm \\
0.05 \mathrm{eB}\end{array}$ & $\begin{array}{l}0.55 \pm \\
0.02 \mathrm{cA}\end{array}$ & $\begin{array}{l}9.34 \pm \\
0.17 \mathrm{eB}\end{array}$ & $\begin{array}{l}14.74 \pm \\
0.25 \mathrm{~dB}\end{array}$ & $\begin{array}{l}7.62 \pm \\
0.15 \mathrm{bcB}\end{array}$ \\
\hline & mean & 5.96 & 23.31 & 57.45 & 2.04 & 0.55 & 9.81 & 17.56 & 8.54 \\
\hline \multirow[t]{3}{*}{ 1538m } & $\begin{array}{l}0 \sim \\
20 \mathrm{~cm}\end{array}$ & $\begin{array}{l}5.55 \pm \\
0.01 \mathrm{bA}\end{array}$ & $\begin{array}{l}33.67 \pm \\
1.53 \mathrm{cA}\end{array}$ & $\begin{array}{l}71.45 \pm \\
0.79 \mathrm{dA}\end{array}$ & $\begin{array}{l}4.12 \pm \\
0.06 \mathrm{cA}\end{array}$ & $\begin{array}{l}0.64 \pm \\
0.01 \mathrm{cA}\end{array}$ & $\begin{array}{l}12.14 \pm \\
0.27 \mathrm{cA}\end{array}$ & $\begin{array}{l}23.83 \pm \\
0.11 \mathrm{eA}\end{array}$ & $\begin{array}{l}5.78 \pm \\
0.09 f A\end{array}$ \\
\hline & $\begin{array}{l}20 \sim \\
40 \mathrm{~cm}\end{array}$ & $\begin{array}{l}5.58 \pm \\
0.01 \mathrm{bA}\end{array}$ & $\begin{array}{l}35.71 \pm \\
2.58 \mathrm{cA}\end{array}$ & $\begin{array}{l}45.25 \pm \\
0.17 \mathrm{~dB}\end{array}$ & $\begin{array}{l}3.97 \pm \\
0.01 \mathrm{bcB}\end{array}$ & $\begin{array}{l}0.63 \pm \\
0.03 \mathrm{bA}\end{array}$ & $\begin{array}{l}11.87 \pm \\
0.16 \mathrm{cA}\end{array}$ & $\begin{array}{l}22.03 \pm \\
0.51 \mathrm{~dB}\end{array}$ & $\begin{array}{l}5.55 \pm \\
0.11 \mathrm{cA}\end{array}$ \\
\hline & mean & 5.57 & 34.69 & 58.35 & 4.05 & 0.64 & 12.01 & 22.93 & 5.67 \\
\hline \multirow[t]{3}{*}{$1744 m$} & $\begin{array}{l}0 \sim \\
20 \mathrm{~cm}\end{array}$ & $\begin{array}{l}5.27 \pm \\
0.00 \mathrm{cA}\end{array}$ & $\begin{array}{l}38.52 \pm \\
2.75 \mathrm{cA}\end{array}$ & $\begin{array}{l}95.13 \pm \\
0.16 \mathrm{cA}\end{array}$ & $\begin{array}{l}4.16 \pm \\
0.03 c A\end{array}$ & $\begin{array}{l}0.66 \pm \\
0.03 \mathrm{cA}\end{array}$ & $\begin{array}{l}9.93 \pm \\
0.05 \mathrm{~dB}\end{array}$ & $\begin{array}{l}34.05 \pm \\
0.26 \mathrm{dA}\end{array}$ & $\begin{array}{l}8.18 \pm \\
0.08 \mathrm{eA}\end{array}$ \\
\hline & $\begin{array}{l}20 \sim \\
40 \mathrm{~cm}\end{array}$ & $\begin{array}{l}5.30 \pm \\
0.01 \mathrm{cA}\end{array}$ & $\begin{array}{l}40.15 \pm \\
2.23 \mathrm{bcA}\end{array}$ & $\begin{array}{l}77.94 \pm \\
0.62 \mathrm{cB}\end{array}$ & $\begin{array}{l}4.09 \pm \\
0.02 \mathrm{bB}\end{array}$ & $\begin{array}{l}0.62 \pm \\
0.01 \mathrm{bcA}\end{array}$ & $\begin{array}{l}10.86 \pm \\
0.19 \mathrm{dA}\end{array}$ & $\begin{array}{l}27.34 \pm \\
11.37 \mathrm{dA}\end{array}$ & $\begin{array}{l}6.67 \pm \\
2.73 \mathrm{cA}\end{array}$ \\
\hline & mean & 5.29 & 39.34 & 86.53 & 4.13 & 0.64 & 10.40 & 30.70 & 7.42 \\
\hline \multirow[t]{3}{*}{ 1970m } & $\begin{array}{l}0 \sim \\
20 \mathrm{~cm}\end{array}$ & $\begin{array}{l}4.86 \pm \\
0.06 \mathrm{~d} A\end{array}$ & $\begin{array}{l}46.23 \pm \\
1.12 \mathrm{bA}\end{array}$ & $\begin{array}{l}95.63 \pm \\
0.21 \mathrm{cA}\end{array}$ & $\begin{array}{l}4.18 \pm \\
0.01 \mathrm{cA}\end{array}$ & $\begin{array}{l}0.68 \pm \\
0.01 \mathrm{cA}\end{array}$ & $\begin{array}{l}10.22 \pm \\
0.22 \mathrm{~dB}\end{array}$ & $\begin{array}{l}54.44 \pm \\
0.74 \mathrm{cA}\end{array}$ & $\begin{array}{l}13.01 \pm \\
0.17 \mathrm{cA}\end{array}$ \\
\hline & $\begin{array}{l}20 \sim \\
40 \mathrm{~cm}\end{array}$ & $\begin{array}{l}4.89 \pm \\
0.02 \mathrm{dA}\end{array}$ & $\begin{array}{l}47.7 \pm \\
0.79 \mathrm{bA}\end{array}$ & $\begin{array}{l}78.34 \pm \\
0.24 \mathrm{cB}\end{array}$ & $\begin{array}{l}3.87 \pm \\
0.05 \mathrm{cB}\end{array}$ & $\begin{array}{l}0.67 \pm \\
0.01 \mathrm{bA}\end{array}$ & $\begin{array}{l}11.11 \pm \\
0.15 \mathrm{~cd} A\end{array}$ & $\begin{array}{l}40.35 \pm \\
0.50 \mathrm{cB}\end{array}$ & $\begin{array}{l}10.42 \pm \\
0.01 b B\end{array}$ \\
\hline & mean & 4.88 & 46.97 & 86.98 & 4.03 & 0.68 & 10.66 & 47.40 & 11.71 \\
\hline \multirow[t]{3}{*}{ 2135m } & $\begin{array}{l}0 \sim \\
20 \mathrm{~cm}\end{array}$ & $\begin{array}{l}4.52 \pm \\
0.01 \mathrm{eA}\end{array}$ & $\begin{array}{l}57.89 \pm \\
4.44 a A\end{array}$ & $\begin{array}{l}215.05 \pm \\
1.51 \mathrm{aA}\end{array}$ & $\begin{array}{l}4.70 \pm \\
0.14 \mathrm{bA}\end{array}$ & $\begin{array}{l}1.02 \pm \\
0.04 \mathrm{aA}\end{array}$ & $\begin{array}{l}13.99 \pm \\
0.65 \mathrm{bA}\end{array}$ & $\begin{array}{l}74.6 \pm \\
0.73 \mathrm{aA}\end{array}$ & $\begin{array}{l}15.86 \pm \\
0.33 \mathrm{bB}\end{array}$ \\
\hline & $\begin{array}{l}20 \sim \\
40 \mathrm{~cm}\end{array}$ & $\begin{array}{l}4.53 \pm \\
0.01 \mathrm{eA}\end{array}$ & $\begin{array}{l}59.49 \pm \\
4.91 \mathrm{aA}\end{array}$ & $\begin{array}{l}121.03 \pm \\
1.46 \mathrm{bB}\end{array}$ & $\begin{array}{l}3.37 \pm \\
0.09 \mathrm{~dB}\end{array}$ & $\begin{array}{l}0.86 \pm \\
0.06 \mathrm{aB}\end{array}$ & $\begin{array}{l}15.22 \pm \\
0.55 \mathrm{bA}\end{array}$ & $\begin{array}{l}71.18 \pm \\
0.51 b B\end{array}$ & $\begin{array}{l}21.11 \pm \\
0.69 a A\end{array}$ \\
\hline & mean & 4.53 & 58.69 & 168.04 & 4.04 & 0.94 & 14.60 & 72.89 & 18.48 \\
\hline \multirow[t]{3}{*}{ 2376m } & $\begin{array}{l}0 \sim \\
20 \mathrm{~cm}\end{array}$ & $\begin{array}{l}4.46 \pm \\
0.01 \mathrm{eA}\end{array}$ & $\begin{array}{l}61.80 \pm \\
2.50 \mathrm{aA}\end{array}$ & $\begin{array}{l}162.81 \pm \\
0.64 b A\end{array}$ & $\begin{array}{l}5.62 \pm \\
0.07 \mathrm{aA}\end{array}$ & $\begin{array}{l}0.86 \pm \\
0.03 \mathrm{bA}\end{array}$ & $\begin{array}{l}17.15 \pm \\
0.16 a \mathrm{~B}\end{array}$ & $\begin{array}{l}104.43 \pm \\
0.73 a A\end{array}$ & $\begin{array}{l}18.6 \pm \\
0.34 \mathrm{aB}\end{array}$ \\
\hline & $\begin{array}{l}20 \sim \\
40 \mathrm{~cm}\end{array}$ & $\begin{array}{l}4.50 \pm \\
0.00 \mathrm{eA}\end{array}$ & $\begin{array}{l}60.36 \pm \\
5.72 a A\end{array}$ & $\begin{array}{l}135.19 \pm \\
0.83 \mathrm{aB}\end{array}$ & $\begin{array}{l}4.27 \pm \\
0.09 \mathrm{aB}\end{array}$ & $\begin{array}{l}0.79 \pm \\
0.02 \mathrm{aB}\end{array}$ & $\begin{array}{l}17.78 \pm \\
0.22 \mathrm{aA}\end{array}$ & $\begin{array}{l}87.09 \pm \\
0.23 \mathrm{aB}\end{array}$ & $\begin{array}{l}20.4 \pm \\
0.36 \mathrm{aA}\end{array}$ \\
\hline & mean & 4.48 & 61.08 & 149 & 4.94 & 0.82 & 17.47 & 95.76 & 19.5 \\
\hline $\begin{array}{l}\text { Differen } \\
\text { Sims va } \\
0.05 \rrbracket a n \\
\text { nitroger }\end{array}$ & er-c & tters in & a sign & $\begin{array}{l}\text { differen } \\
\text { the sa } \\
\text { in diffe } \\
\text { SOC, s }\end{array}$ & $\begin{array}{l}05) \text { as d } \\
\text { layer; Di } \\
\text { I layers. } \\
\text { lic carbc }\end{array}$ & $\begin{array}{l}\text { ned by } \\
\text { capita } \\
\text { soil wa }\end{array}$ & $\begin{array}{l}\text { test am } \\
\text { indicat } \\
\text { tent; OM }\end{array}$ & $\begin{array}{l}\text { nizospher } \\
\text { gnificant } \\
\text { hic matte }\end{array}$ & $\begin{array}{l}\text { of } A \text {. crena } \\
\text { ences } \square P< \\
\text { otal }\end{array}$ \\
\hline
\end{tabular}

significantly with elevation; however, there was no significant difference in SWC with increasing depth at any of the sites. Soil OM content increased with elevation from 1,257 to 2,135 m a.s.l., where it reached a maximum of $215.05 \mathrm{~g} \cdot \mathrm{kg}^{-1}$, which was slightly higher than the soil $\mathrm{OM}$ of the site at the highest elevation. At all sites, soil OM decreased with depth. The values of several soil chemical properties varied with altitude. The variation in TP content was consistent with that of OM, and the TN and TK content increased with elevation. At the highest elevations, the concentration of TN was 2.42 times higher, and the concentration of TK 1.78 times higher than those at the lowest elevations $(P<0.05)$. The same trends in vertical distribution were identified for SOC and TN, and these soil nutrients decreased with depth at all elevations. In contrast, TK increased with depth at higher elevations. The $\mathrm{C} / \mathrm{N}$ ratio was lowest at 1,538 $\mathrm{m}$ a.s.I., after which it increased, reaching its highest value at 2,376 $\mathrm{m}$ a.s.l.; it showed a clear increase with elevation.

Soil absolute enzyme activity and specific enzyme activity per unit soil organic carbon 
There were significant differences in enzyme activity with respect to elevation and to soil physicochemical properties. The activity of URE and SAC tended to first increase and then decrease with elevation. Both these enzymes showed maximum activity in the 0 to 20 -cm layer at the $2,135 \mathrm{~m}$ a.s.I. site (URE: $10.18 \mathrm{mg} \cdot \mathrm{g}^{-1}$ and SAC: $4.89 \mathrm{mg} \cdot \mathrm{g}^{-1}$ ) (Fig. 2A, B). The activity of AMY, POD, and CAT showed significantly positive correlations with elevation. All three of these enzymes showed maximum activity in the $0-20 \mathrm{~cm}$ layer at the highest elevation (AMY: $7.69 \mathrm{mg} \cdot \mathrm{g}^{-1}, \mathrm{POD}: 474.97$

$\mathrm{mg} \cdot \mathrm{g}^{-1}$, and CAT: $3.02 \mathrm{ml} \cdot \mathrm{g}^{-1}$ ), and minimum activity at the lowest elevation (Fig. $2 \mathrm{C}-\mathrm{E}$ ). All the soil enzymes showed a significant decrease in activity with soil depth. The SOCE of URE, SAC, AMY, POD, and CAT in the $0-20 \mathrm{~cm}$ layer increased and then decreased along the altitudinal gradient (Fig. 2a-e). The POD/SOC values along the altitudinal gradient were significantly different and peaked at the 1,744 $\mathrm{m}$ a.s.I. site, whereas the URE/SOC and SAC/SOC values peaked at the 1,970 $\mathrm{m}$ a.s.I. site, and the AMY/SOC and CAT/SOC values peaked at the 1,538 m a.s.I. site. Overall, SOCE decreased with soil depth at different elevations. Based on the Pearson correlation analyses, soil physicochemical

properties (except for soil $\mathrm{pH}$ ) and enzyme activity were strongly positively correlated with elevation. Significant negative correlations with elevation were observed for AMY/SOC, POD/SOC, and CAT/SOC $(P<0.01)$, whereas URE/SOC and SAC/SOC showed no significant correlations with elevation (Supplementary data, Table S1).

Correlations between soil physicochemical properties and enzyme activity

Principal Component Analysis was used to identify the relationships between the soil properties and enzyme activity (Table 3). Two principal factors had a significant impact on the variance of the variables (88.06\%). It was found that the first principal component (PC1) correlated negatively with soil $\mathrm{pH}$ but positively with the other soil properties. The value of the second principal component (PC2) (10.94\%) mainly revealed a positive correlation with URE and SAC and a negative correlation with TK, pH, and SOC. In addition, the RDA ordination biplot in Fig. 3A indicated a correlation between soil properties and enzyme activity and SOCE along the elevation gradient. Axis 1 and axis 2 of the RDA explained $99.6 \%$ and $0.03 \%$ of the total variation, respectively, and the eight environmental factors associated with the first two axes could explain $99.7 \%$ of the soil enzyme activity characteristics. This was mainly determined by the first axis and could elucidate the correlation between soil enzyme activity and environmental factors. The activity of SAC, CAT, AMY, POD, and URE was positively correlated with TN, TP, TK, SOC, OM, SWC, and C/N, whereas enzyme activity was negatively correlated with pH. There was a negative correlation between URE/SOC, AMY/SOC, CAT/SOC, and POD/SOC activity and soil nutrients. Among them, the OM content was the dominant factor affecting the activity of CAT and AMY, and the SOC content mainly affected the POD activity. 
Table 3

Principal component analysis between soil properties and enzyme activity, plant physiological attributes

\begin{tabular}{|c|c|c|c|c|c|}
\hline \multirow[t]{2}{*}{ Elements } & \multicolumn{2}{|l|}{ Soil properties } & \multirow[t]{2}{*}{ Elements } & \multicolumn{2}{|c|}{ Plant physiology } \\
\hline & PC1(77.12\%) & PC2 (10.94\%) & & PC1 (60.97\%) & PC2 (21.73\%) \\
\hline Swc & 0.260 & 0.059 & Chla & 0.325 & 0.189 \\
\hline $\mathrm{pH}$ & -0.261 & -0.128 & Chlb & 0.340 & -0.031 \\
\hline $\mathrm{OM}$ & 0.254 & -0.02 & Chlt & 0.337 & 0.114 \\
\hline TN & 0.188 & -0.007 & $a / b$ & 0.043 & 0.548 \\
\hline TP & 0.247 & 0.003 & ANT & 0.220 & 0.221 \\
\hline TK & 0.233 & -0.321 & PRO & 0.290 & 0.260 \\
\hline SOC & 0.263 & -0.101 & SS & 0.331 & -0.089 \\
\hline $\mathrm{C} / \mathrm{N}$ & 0.255 & -0.063 & ST & 0.228 & 0.344 \\
\hline URE & 0.218 & 0.400 & SOD & 0.217 & -0.346 \\
\hline SAC & 0.258 & 0.150 & POD & 0.277 & -0.170 \\
\hline AMY & 0.255 & 0.033 & CAT & 0.264 & -0.296 \\
\hline POD & 0.256 & 0.009 & PPO & 0.251 & -0.412 \\
\hline CAT & 0.252 & 0.043 & PAL & 0.336 & 0.051 \\
\hline
\end{tabular}

Physiological responses of $A$. crenata var. bicolor associated with the altitudinal gradient

The thirteen physiological variables of $A$. crenata var. bicolor showed different sensitivities to the altitudinal gradient (Table 4). The Pearson correlation analyses indicated that plant physiological properties were positively correlated with elevation (Supplementary data, Table S2). The chlorophyll and anthocyanin content increased with elevation, whereas the chlorophyll $\mathrm{a} / \mathrm{b}$ ratio first increased and then decreased (2,376 $\mathrm{m}$ a.s.l.; 1.60); however, the anthocyanin content did not differ significantly $(P>0.05)$. The protein content in the leaves of $A$. crenata var. bicolor increased significantly with elevation, peaking at 2,135 $\mathrm{m}$ a.s.l. and decreasing at 2,376 $\mathrm{m}$ a.s.l., but not significantly. The soluble sugar and starch content of the leaves increased along the altitudinal gradient, reaching maximum amounts at 2,376 $\mathrm{m}$ a.s.l. (12.38\%) and at $1,970 \mathrm{~m}$ a.s.I. (8.97\%), respectively, and then gradually decreased. The activity of SOD, POD, CAT, PPO, and PAL was consistent along the altitudinal gradient. The activity of five of the enzymes was relatively high at the lowest altitude (1,257 $\mathrm{m}$ a.s.l.), which might be related to frequent anthropogenic actions at this elevation. At higher elevations, SOD, CAT, and PPO activities decreased and then increased, peaking at 2,376 $\mathrm{m}$ a.s.I., whereas the POD and PAL activity decreased slightly, but not significantly, compared to that at the 2,135 $\mathrm{m}$ a.s.I. elevation. 
Table 4

Plant physiological attributes of $A$. crenata Sims var. bicolor with elevations

\begin{tabular}{|c|c|c|c|c|c|c|c|c|c|c|c|c|c|}
\hline Altitude & Chla & Chlb & Chlt & $a / b$ & ANT & PRO & SS & ST & SOD & POD & CAT & PPO & PAL \\
\hline $1257 m$ & $\begin{array}{l}7.84 \\
\pm \\
0.02 d\end{array}$ & $\begin{array}{l}4.75 \\
\pm \\
0.01 d\end{array}$ & $\begin{array}{l}12.58 \\
\pm \\
0.03 d\end{array}$ & $\begin{array}{l}1.65 \\
\pm \\
0.00 \mathrm{~b}\end{array}$ & $\begin{array}{l}20.56 \\
\pm \\
0.33 a\end{array}$ & $\begin{array}{l}6.97 \\
\pm \\
0.01 d\end{array}$ & $\begin{array}{l}9.87 \\
\pm \\
0.02 d\end{array}$ & $\begin{array}{l}4.16 \\
\pm \\
0.07 f\end{array}$ & $\begin{array}{l}459.84 \\
\pm \\
9.81 a b\end{array}$ & $\begin{array}{l}69.27 \\
\pm 5.77 b\end{array}$ & $\begin{array}{l}14.02 \\
\pm \\
0.89 \mathrm{~b}\end{array}$ & $\begin{array}{l}256.85 \\
\pm 1.15 b\end{array}$ & $\begin{array}{r}127.88 \\
\pm 8.46 b\end{array}$ \\
\hline $1538 m$ & $\begin{array}{l}7.87 \\
\pm \\
0.01 d\end{array}$ & $\begin{array}{l}4.74 \\
\pm \\
0.00 d\end{array}$ & $\begin{array}{l}12.62 \\
\pm \\
0.01 d\end{array}$ & $\begin{array}{l}1.66 \\
\pm \\
0.00 \mathrm{~b}\end{array}$ & $\begin{array}{l}20.84 \\
\pm \\
0.19 a\end{array}$ & $\begin{array}{l}10.54 \\
\pm \\
0.17 c\end{array}$ & $\begin{array}{l}9.99 \\
\pm \\
0.03 d\end{array}$ & $\begin{array}{l}5.00 \\
\pm \\
0.03 e\end{array}$ & $\begin{array}{l}452.19 \\
\pm \\
21.57 b\end{array}$ & $\begin{array}{l}39.00 \\
\pm 1.12 \mathrm{c}\end{array}$ & $\begin{array}{l}7.43 \\
\pm \\
1.02 \mathrm{c}\end{array}$ & $\begin{array}{l}181.95 \\
\pm 1.54 \mathrm{~d}\end{array}$ & $\begin{array}{r}123.96 \\
\pm 1.19 b\end{array}$ \\
\hline $1744 m$ & $\begin{array}{l}11.13 \\
\pm \\
0.03 c\end{array}$ & $\begin{array}{l}5.84 \\
\pm \\
0.01 c\end{array}$ & $\begin{array}{l}16.96 \\
\pm \\
0.03 c\end{array}$ & $\begin{array}{l}1.90 \\
\pm \\
0.00 a\end{array}$ & $\begin{array}{l}20.95 \\
\pm \\
0.13 a\end{array}$ & $\begin{array}{l}11.61 \\
\pm \\
0.57 b\end{array}$ & $\begin{array}{l}10.53 \\
\pm \\
0.01 \mathrm{c}\end{array}$ & $\begin{array}{l}5.70 \\
\pm \\
0.01 d\end{array}$ & $\begin{array}{l}437.06 \\
\pm \\
41.22 b\end{array}$ & $\begin{array}{l}27.09 \\
\pm 1.08 \mathrm{c}\end{array}$ & $\begin{array}{l}5.04 \\
\pm \\
0.65 d\end{array}$ & $\begin{array}{l}132.25 \\
\pm 2.39 \mathrm{e}\end{array}$ & $\begin{array}{l}134.05 \\
\pm \\
15.22 b\end{array}$ \\
\hline $1970 m$ & $\begin{array}{l}11.11 \\
\pm \\
0.02 c\end{array}$ & $\begin{array}{l}5.84 \\
\pm \\
0.01 c\end{array}$ & $\begin{array}{l}16.94 \\
\pm \\
0.03 c\end{array}$ & $\begin{array}{l}1.91 \\
\pm \\
0.01 \mathrm{a}\end{array}$ & $\begin{array}{l}21.02 \\
\pm \\
0.12 a\end{array}$ & $\begin{array}{l}12.48 \\
\pm \\
0.54 b\end{array}$ & $\begin{array}{l}10.59 \\
\pm \\
0.12 c\end{array}$ & $\begin{array}{l}8.97 \\
\pm \\
0.04 a\end{array}$ & $\begin{array}{l}428.95 \\
\pm \\
27.36 b\end{array}$ & $\begin{array}{l}74.77 \\
\pm 2.84 b\end{array}$ & $\begin{array}{l}14.45 \\
\pm \\
0.27 \mathrm{~b}\end{array}$ & $\begin{array}{l}205.63 \\
\pm 7.97 c\end{array}$ & $\begin{array}{l}155.67 \\
\pm \\
4.77 a b\end{array}$ \\
\hline $2135 m$ & $\begin{array}{l}13.11 \\
\pm \\
0.04 a\end{array}$ & $\begin{array}{l}6.89 \\
\pm \\
0.14 \mathrm{~b}\end{array}$ & $\begin{array}{l}19.99 \\
\pm \\
0.18 \mathrm{~b}\end{array}$ & $\begin{array}{l}1.90 \\
\pm \\
0.03 a\end{array}$ & $\begin{array}{l}21.12 \\
\pm \\
0.32 a\end{array}$ & $\begin{array}{l}13.98 \\
\pm \\
0.11 \mathrm{a}\end{array}$ & $\begin{array}{l}11.28 \\
\pm \\
0.09 \mathrm{~b}\end{array}$ & $\begin{array}{l}7.59 \\
\pm \\
0.08 \mathrm{~b}\end{array}$ & $\begin{array}{l}486.87 \\
\pm \\
15.67 a b\end{array}$ & $\begin{array}{l}103.42 \\
\pm \\
12.41 a\end{array}$ & $\begin{array}{l}15.38 \\
\pm \\
0.27 \mathrm{~b}\end{array}$ & $\begin{array}{l}265.84 \\
\pm 2.75 b\end{array}$ & $\begin{array}{l}175.83 \\
\pm 2.98 a\end{array}$ \\
\hline $2376 m$ & $\begin{array}{l}12.76 \\
\pm \\
0.02 \mathrm{~b}\end{array}$ & $\begin{array}{l}7.96 \\
\pm \\
0.02 a\end{array}$ & $\begin{array}{l}20.72 \\
\pm \\
0.03 a\end{array}$ & $\begin{array}{l}1.60 \\
\pm \\
0.00 \mathrm{c}\end{array}$ & $\begin{array}{l}21.10 \\
\pm \\
0.35 a\end{array}$ & $\begin{array}{l}13.43 \\
\pm \\
0.18 a\end{array}$ & $\begin{array}{l}12.38 \\
\pm \\
0.17 a\end{array}$ & $\begin{array}{l}6.45 \\
\pm \\
0.09 c\end{array}$ & $\begin{array}{l}520.20 \\
\pm \\
12.25 \mathrm{a}\end{array}$ & $\begin{array}{l}91.73 \\
\pm 3.23 \mathrm{a}\end{array}$ & $\begin{array}{l}19.59 \\
\pm \\
0.72 a\end{array}$ & $\begin{array}{l}394.43 \\
\pm 6.14 a\end{array}$ & $\begin{array}{l}173.07 \\
\pm \\
12.76 \mathrm{a}\end{array}$ \\
\hline
\end{tabular}

Different lower-case letters indicates a significant difference $(P<0.05)$ among physiological attributes of $A$. crenata Sims var. bicolor determined by one-way ANOVA.

Chla, chlorophyll a; Chlb, chlorophyll b; Chlt, total chlorophyll; a/b, Chla: Chlb; ANT, anthocyanin; PRO, protein; SS, soluble sugar; ST, starch content; SOD, superoxide dismutase; POD, peroxidase; CAT, catalase; PPO, polyphenol oxidase; PAL, phenylalanine ammonialyase.

Principal component analysis of the plant traits showed that $82.70 \%$ of the total variance was explained by the first two principal components (Table 3). Of the total variance, PC1 explained $60.97 \%$ and the main explanatory variables were chlorophyll, sugar content, and PAL. Similarly, PC2 explained $21.73 \%$ of the variance and was largely related to a/b ratio, PPO, starch content, and SOD. In this study, the response of $A$. crenata var. bicolor leaf physiological traits to soil factors was analyzed by RDA. Axis 1 and axis 2 explained $87.7 \%$ and $4.7 \%$ of the total variation, respectively (Fig. 3B). The cumulative variation was $95.1 \%$, which could reflect the relationships between plant physiological traits and soil factors. Axis 1 of the RDA reflected a positive correlation between soil TN, TP, TK, OM, SOC, SWC, C/N, and the physiological traits of $A$. crenata var. bicolor leaves, but the correlation of TN was relatively weak. Axis 2 of the RDA mainly reflected the negative correlation between physiological traits and $\mathrm{pH}$. The RDA ordination biplot showed the different responses of physiological traits to soil factors. The OM content and TP were the dominant factors affecting the activity of PAL, whereas SWC affected the Chl t content, the $\mathrm{C} / \mathrm{N}$ ratio was related to the $\mathrm{POD}$ activity, the SOC content mainly affected the Chl b content and CAT activity, and the content of TK mainly affected SOD activity and SS synthesis and accumulation.

\section{Discussion}

On forested mountains, altitude represents a complex gradient related to the function of the forest ecosystem and soil nutrient limitations as altitude changes. Carbon and nitrogen play an important role in both climate change and plant growth, and dynamic changes in soil carbon and nitrogen content could indicate human disturbance in forest ecosystems, thus the quantification of these substances could provide insights into interactions among drivers, pressures, states, and responses to environmental change. Several studies have reported that the larger the stock of SOC and TN, the greater the contribution to climate change (Dar and Somaiah 2015; Gebrehiwot et al. 2018; Parras- Alcántara et al. 2015). In tropical forests or dry-hot valley regions, SOC, TN, and TP increased with elevation (Nottingham et al. 2015; Xiao et al. 2018), a trend which was mainly associated with elevation-linked variation in precipitation and temperature, and restricted the decomposition of plant litter and the cycling of OM (Du et al. 2014). On the Gaoligong Mountain we observed that soil OM accumulates on the forest floor and that TN, TP, and TK in A. crenata var. bicolor rhizosphere soil, sampled under shrubs at different altitudes, increased significantly with elevation, a finding consistent with that of Yu et al. (2014). Climate changes occur along the entire altitudinal gradient on the Gaoligong Mountain; however, anthropogenic disturbances, excavation, and livestock trampling are most serious at lower altitudes, causing soil compaction and decreasing the available oxygen and soil nutrient and SOC content. A high $\mathrm{C} / \mathrm{N}$ ratio at high altitude indicated that litter decomposition decreased in correlation with the production and long-term accumulation of SOC content which can be more affected by altitude than by soil texture (Zinn et al. 2018). McKinley et al. (2008) also indicated that shrub-type plants could increase carbon 
availability and contribute to increased soil quality. Therefore, differences in physicochemical properties appear to be correlated with elevation and above-ground vegetation litter.

Pearson correlation analysis showed a strong positive correlation of enzyme activity with SWC, OM, SOC, and other nutrients, a finding consistent with Moghimian et al. (2017), and there were significant differences in enzyme activity with altitude (Supplementary data, Table S1). The lower enzyme activity at lower altitudes could be linked to reduced secretion of root and organic residues together with the anthropogenic exploitation and livestock trampling of $A$. crenata var. bicolor, which is detrimental to the transformation and accumulation of soil enzymes. When the significantly high soil enzyme activity at high altitude was compared to that at low altitude, a decreasing trend with increasing soil depth was observed. In relation to vegetation type, there was a descending order of enzyme activity from mid-mountain humid evergreen broad-leaved forest to semi-humid evergreen broad-leaved forest to monsoon evergreen broad-leaved forest to tropical monsoon forest. Among the enzymes assayed, URE, SAC, AMY, POD, and CAT activity mediated soil OM degradation, transformation, and mineralization, and increased with the increase in organic residues. Therefore, it appeared that OM content greatly affected physicochemical properties and soil nutrients, the distribution of which might explain changes in enzyme activity in the soil profile. Among the enzymes, SAC was the most sensitive, and SAC activity was 5.34 and 7.38 times higher in the topsoil and subsoil layers, respectively, at high altitude $(2,376 \mathrm{~m})$ than at low altitude $(1,257 \mathrm{~m})$. It is unclear whether SAC sensitivity was caused by the activity of the enzyme itself or by the soil microbial biomass and OM (Wang et al. 2012). Enzyme activity can also significantly affect the decomposition of soil OM and thus, influence the storage and cycling of carbon and nitrogen (Cenini et al. 2016). Soil enzyme activity and SOC decreased with soil depth, but the rate of decrease in enzyme activity was higher than that for SOC, which might be caused by the slow decrease of SOC. The SOC/TN ratios of the soils from A. crenata var. bicolor habitats ranged widely (5.55-20.40). Consequently, the mineralization of the organic substance may have been slowed. According to the Pearson correlation analysis, $\mathrm{C} / \mathrm{N}$ showed no significant correlation with $\mathrm{TN}$, and was mainly affected by SOC (Supplementary data, Table S1). Purahong et al. (2016) found that the most important factors affecting soil enzyme activity were tree species traits, followed by soil properties. Ayres et al. (2009) also showed that plants have species-specific effects on soil properties. This finding reflects that tree species may affect soil physicochemical properties in forest ecosystems and alter the quantity and quality of leaf litter via root activities.

Although soil absolute enzyme activity is sensitive to environmental changes, measures of specific enzyme activity provided more ecological information and responded more clearly to changes in altitude than the absolute enzyme activity (Raiesi and Beheshti 2014; Xiao et al. 2018). Our results indicate that the altitudinal gradient affected the SOCE of $A$. crenata var. bicolor habitats. This may be because the destruction of soil structure decreased with elevation, which might have increased soil enzyme activity by increasing the potential for contact between SOC and soil enzymes. Similarly, OM may also have influenced SOCE at different elevations. The high SOCE may have resulted from the low OM content in the soil, which was also related to other factors such as vegetation and enzyme species (Wang et al. 2012).

In this study, the changes in SOCE (URE/SOC and SAC/SOC) were similar to that of soil absolute enzyme activity (Fig. 2a, b), initially increasing and then decreasing, whereas AMY/SOC, POD/SOC, and CAT/SOC showed responses opposite to those of soil absolute enzyme activity, decreasing with soil depth. This result shows that topsoil nutrients are mainly affected by plant litter and root exudates, and that deeper soil might be restricted by a lower soil nutrient and microbial biomass (Stone et al. 2014). In addition, SOCE is significantly correlated with changes in soil aggregates (Dungait et al. 2012), and SOC change could be used as a response index to reflect vegetation restoration (Xu et al. 2020). Therefore, studying SOCE may play a significant role in studying the interactions of soil nutrients and enzymes, and it may serve as a better tool for elucidating soil enzyme activity. Owing to a lack of temperature and precipitation data along the altitudinal gradient on Gaoligong Mountain, we were not able to analyze the effects of these two environmental factors on the distribution pattern of enzyme activity and SOC. However, the effect was deduced indirectly by correlation analysis with altitude and was found to be greater in the upper than in the lower soil layer.

Regarding the similar stand densities at different altitudes, a more open canopy and higher penetration of light were found with increasing altitude. Chlorophyll content is positively associated with photosynthetic capacity, nutrient condition, and elevation, which are sensitive to environmental factors. Light intensity, particularly, can decrease chlorophyll content by slowing chlorophyll synthesis or accelerating decomposition (Hazrati et al. 2016). In the present study, the Chl t increased with altitude, which might reflect the accumulation of photosynthetic pigment under restrictive growth conditions. However, the $\mathrm{Chl}$ a: $\mathrm{Chl} b$ ratio was significantly higher at 1,744-2,135 $\mathrm{m}$ a.s.l. in the humid evergreen broad-leaved forest than at other altitudes, indicating that this altitude range may be more suitable for the growth of A. crenata var. bicolor. The $\mathrm{Chl}$ a: $\mathrm{Chl} b$ ratio decreased to its lowest level at 2,376 $\mathrm{m}$ a.s.l., which represents the growth tolerance extreme of A. crenata var. bicolor on the Gaoligong Mountain. This finding may be related to a decrease in $\mathrm{Chl}$ a and photosynthesis and an increase in photoinhibition as an adaptive response to avoid the damage caused by environmental stress. In addition, the upper and lower canopy leaves have different acclimation adaptations. The morphological, physiological, and biochemical characteristics of lower canopy leaves 
were modified along the elevation gradient to cope with changing conditions (Rajsnerová et al. 2014). Such leaf adaptation plasticity could cause significant variation in the photosynthesis of shrub canopies and its contribution to the overall physiological balance of the plant.

Soluble protein and sugar are considered important osmotic regulators during abiotic stress and can be induced by multiple environmental factors, either directly or indirectly. In this study, several carbohydrates were correlated with altitude, and the protein content in A. crenata var. bicolor leaves increased and leveled off with the increase in altitude, where the average content at 2,376 $\mathrm{m}$ a.s.l. was slightly lower than that at 2,135 $\mathrm{m}$ a.s.I. but not significantly different. Soluble sugar accumulation increased with elevation, and the maximum sugar content (12.38\%) was recorded in leaves at the highest altitude. Starch content peaked at 1,970 $\mathrm{m}$ a.s.l., and then decreased, which might reflect the conversion of starch to sucrose at high altitude and a response to coping with low temperatures. Sugar replaces water molecules and establishes hydrogen bonds with lipid molecules in plants at high altitudes and acts as a defense mechanism, thus protecting cell membrane structures from cold stress (Basu et al. 2007; Ruelland et al. 2009). Therefore, carbohydrate accumulation will stabilize cell membranes, increase cellular liquid concentrations, reduce osmotic potential, and directly control and activate the resistance genes and/or metabolic pathways linked to different environmental pressures (Kosová et al. 2018).

Frequent human activities and excavation at low altitude can enhance enzyme activity in leaves. The activity of SOD, POD, CAT, PPO, and PAL was significantly positively correlated with altitude, which suggests that the plant has a different reactive oxygen species scavenging capacity at different altitudes. The RDA revealed the different physiological responses of $A$. crenata var. bicolor along the altitudinal gradient, which indicated the important role played by physiology in adaptation to the environments on the Gaoligong Mountain. The variation in species habitat was primarily controlled by elevation and soil conditions, whereas the altitudinal gradient negatively affected the adaptive mechanisms and survival strategies of $A$. crenata var. bicolor on the Gaoligong Mountain. In this study, we did not consider interaction factors like species composition, diffusion path, biological interaction, and productivity that influence the structure and composition of forests.

\section{Conclusions}

The current investigation suggests that altitudinal gradient significantly affects soil properties and the plant physiological attributes of $A$. crenata var. bicolor on the Gaoligong Mountain, which can be used as an index for understanding plant responses to altitudinal change. Soil nutrient content and enzyme activity increased with altitude and decreased with soil depth. The physiological compound content in the leaves was greater at high altitude, including more photosynthetic pigments and carbohydrates. Enzyme activity in the leaves increased in response to oxidative stress, most likely due to adaptive strategies to cope with environmental and other abiotic factors which might induce cold damage and limit the growth range of $A$. crenata var. bicolor. At low altitude, anthropogenic disturbances, excavation, and livestock trampling also increased enzyme activity in the leaves. In addition, plants can alter certain physicochemical characteristics of the soil, which depend on the vegetation types and residues along the altitudinal gradient. Through plant habitat range assessment, and by investigating whether the underlying physiological mechanisms of understory plants are a response to altitude or soil properties, habitat and environmental adaptation was revealed. Further studies should investigate how plant photosynthetic characteristics and phytohormones are affected by altitudinal gradients.

\section{Abbreviations}

OM, organic matter; SWC, soil water content; SOC, soil organic carbon; TN, total nitrogen; TP, total phosphorus; TK, total potassium; URE, soil urease; SAC, saccharase; AMY, amylase; CAT, catalase; POD, peroxidase; SOCE, specific enzyme activity per unit soil organic carbon; Chl a, chlorophyll a; Chl b, chlorophyll b; Chl t, total chlorophyll; ANT, anthocyanin; PRO, protein; SS, soluble sugar; ST, starch content; SOD, superoxide dismutase; PPO, polyphenol oxidase; PAL, phenylalanine ammonia-lyase; RDA, Redundancy analysis.

\section{Declarations}

\section{Authorship contribution statement}

XMA: Resource investigation, conceptualization, methodology, data analysis, software, writing- original manuscript, visualization, YL: ecological sampling, software, validation, supervision, revision, HX, CBZ: resources, methodology, execution, data curation. All authors read and approved the final manuscript.

\section{Acknowledgments}

We would like to thank Chao Wang for providing valuable comments for the manuscript. This work was supported by the Doctoral Research Fund project of Southwest Forestry University (CN) [grant number 111806]. We would like to thank Editage (www.editage.cn) for English 
language editing.

\section{Declaration of competing interests}

The authors declare that they have no known competing financial interests or personal relationships that could have appeared to influence the work reported in this paper.

\section{References}

Ahmad KS, Hameed M, Fatima, S., Ashraf M, Ahmad F, Naseer M, Akhtar N (2016) Morpho-anatomical and physiological adaptations to high altitude in some Aveneae grasses from Neelum Valley, Western Himalayan Kashmir. Acta Physiol Plant 38, 93.

https://doi.org/10.1007/s11738-016-2114-x

Ayres E, Steltzer H, Berg S, Wallenstein MD, Simmons BL, Wall DH (2009) Tree Species Traits Influence Soil Physical, Chemical, and Biological Properties in High Elevation Forests. PLOS ONE. https://doi.org/10.1371/journal.pone.0005964

Bao D (2008) Soil agrochemical analysis, China Agricultural Press, Beijing, China.

Basu PS, Berger JD, Turner NC, Chaturvedi SK, Ali M, Siddique, KHM (2007) Osmotic adjustment of chickpea (Cicer arietinum) is not associated with changes in carbohydrate composition or leaf gas exchange under drought. Ann Appl Biol 150, 217-225.

https://doi.org/10.1111/j.1744-7348.2007.00119.x

Brzezińska M, Rafalski P, Włodarczyk T, Szarlip P, Brzeziński K (2011) How much oxygen is needed for acetylene to be consumed in soil? J Soils Sediments 11, 1142-1154. https://doi.org/10.1007/s11368-011-0419-3

Burns RG, Deforest JL, Marxsen J, Sinsabaugh RL, Stromberger, ME, Wallenstein MD, Weintraub MN, Zoppini A (2013) Soil enzymes in a changing environment: current knowledge and future directions. Soil Biol Biochem 58, 216-234.

https://doi.org/10.1016/j.soilbio.2012.11.009

Byars SG, Papst W, Hoffmann AA (2007) Local adaptation and cogradient selection in the alpine plant, Poa hiemata, along a narrow altitudinal gradient. Evolution 61, 2925-2941. https://doi.org/10.1111/j.1558-5646.2007.00248.x

Cenini VL, Fornara DA, McMullan G, Ternan N, Carolan R, Crawley MJ, Clement JC, Lavorel S (2016) Linkages between extracellular enzyme activities and the carbon and nitrogen content of grassland soils. Soil Biol Biochem 96, 198-206.

https://doi.org/10.1016/j.soilbio.2016.02.015

Chen BX, Zhang XZ, Tao J, Wu JS, Wang JS, Shi PL, Zhang YJ, Yu CQ (2014)The impact of climate change and anthropogenic activities on alpine grassland over the Qinghai-Tibet Plateau. Agr Forest Meteorol 189, 11-18. https://doi.org/10.1016/j.agrformet.2014.01.002

Chen RS, Han CT, Liu JF, Yang Y, Liu ZW, Wang L, Kang E (2018) Maximum precipitation altitude on the northern flank of the Qilian Mountains, northwest China. Hydrol Res 49, 1696-1710. https://doi.org/10.2166/nh.2018.121

Chodak M, Klimek B, Azarbad H, Jaźwa M (2015) Functional diversity of soil microbial communities under Scots pine, Norway spruce, silver birch and mixed boreal forest. Pedobiologia 58: 81-88. https://doi.org/10.1016/j.pedobi.2015.04.002

Cui GX, Wei XH, Degen AA, Wei X, Zhou JW, Ding LM, Shang ZH, Liu SM, Long RJ (2016) Troloxequivalent antioxidant capacity and composition of five alpine plant species growing at different elevations on the Qinghai-Tibetan Plateau. Plant Ecol Divers 9, 387-396. http://doi.org/10.1080/17550874.2016.1261952

Dar JA, Somaiah S (2015) Altitudinal variation of soil organic carbon stocks in temperate forests of Kashmir Himalayas, India. Environ Monit Assess https://doi.org/10.1007/s10661-014-4204-9

De Feudis M, Cardelli V, Massaccesi L, Bol R, Willbold S, Cocco S, Agnelli A (2016) Effect of beech (Fagus sy/vatica L.) rhizosphere on phosphorous availability in soils at different altitudes (Central Italy). Geoderma 276, 53-63. http://doi.org/10.1016/j.geoderma.2016.04.028

De Frenne P, Graae BJ, Rodríguez-Sanchez F, Kolb A, Chabrerie O, Decocq G, De Kort H, De Schrijver A, Diekmann M, Eriksson O, Gruwez R, Hermy M, Lenoir J, Plue J, Coomes DA, Verheyen K (2013) Latitudinal gradients as natural laboratories to infer Species' responses to temperature. J Ecology 101, 784-795. https://doi.org/10.1111/1365-2745.12074 
Du BM, Kang HZ, Pumpanen J, Zhu PH, Yin S, Zou Q, Wang Z, Kong FQ, Liu CJ (2014) Soil organic carbon stock and chemical composition along an altitude gradient in the Lu shan Mountain, subtropical China. Ecol Res 29, 433-439. https://doi.org/10.1007/s11284-014-1135-4

Dungait JAJ, Hopkins DW, Gregory AS, Whitmore AP (2012) Soil organic matter turnover is governed by accessibility not recalcitrance. Glob Change Biol 18, 1781-1796. https://doi.org/10.1111/j.1365-2486.2012.02665.x

Gebrehiwot K, Desalegn T, Woldu Z, Demissew S, Teferi E (2018) Soil organic carbon stock in Abune Yosef afroalpine and sub-afroalpine vegetation, northern Ethiopia. Ecol Proc https://doi.org/10.1186/s13717-018-0117-9

Guan SY (1986) Soil enzyme and its research methods, Agricultural Press, China, 260-346.

Hazrati S, Tahmasebi-Sarvestani Z, Modarres-Sanavy SAM, Mokhtassi-Bidgoli A, Nicola S (2016) Effects of water stress and light intensity on chlorophyll fluorescence parameters and pigments of Aloe vera L. Plant Physiol and Bioch 106,141-148.

https://doi.org/10.1016/j.plaphy.2016.04.046

Hedo de Santiago J, Lucas-Borja M E, Wic-Baena C, Andrés-Abellán M, De las Heras J (2014) Effects of thinning and induced drought on microbiological soil properties and plant species diversity at dry and semiarid locations. Land Degrad Dev 27, $1151-1162$.

https://doi.org/10.1002/ldr.2361

Herold N, Schöning I, Gutknecht J, Alt F, Boch S, Müller J, Oelmann Y, Socher SA, Wilcke W, Wubet T, Schrumpf M (2014) Soil property and management effects on grassland microbial communities across a latitudinal gradient in Germany. Appl Soil Ecol 73, 41-50.

https://doi.org/10.1016/j.apsoil.2013.07.009

Kim HW, Kim JB, Cho SM, Chung MN, Lee YM, Chu S M, Che JH, Kim SN, Kim SY, Cho YS, Kim JH, Park HJ, Lee DJ (2011) Anthocyanin Changes in the Korean Purple-Fleshed Sweet Potato, Shinzami, as affected by steaming and baking. Food Chem 130, $966-972$.

https://doi.org/10.1016/j.foodchem.2011.08.031

Kitajima K Fox AM, Sato T, Nagamatsu D (2006) Cultivar selection prior to introduction may increase invasiveness: evidence from Ardisia crenata. Biol Invasions 8, 1471-1482. https://doi.org/10.1007/s10530-005-5839-9

Kobayashi H, de Mejía E (2005) The genus Ardisia: a novel source of health-promoting compounds and phytopharmaceuticals. J Ethnopharmacol 96, 347-354. https://doi.org/10.1016/j.jep.2004.09.037

Körner C (2007) The use of 'altitude' in ecological research. Trends Ecol Evol 22, 569-574. https://doi.org/10.1016/j.tree.2007.09.006

Kosová K, Vítámvás P, Urban MO, Práil IT, Renaut J (2018) Plant abiotic stress proteomics: The major factors determining alterations in cellular proteome. Front Plant Sci https://doi.org/10.3389/fpls.2018.00122

Li HS (2000) Theory and Techniques of Plant Physiology and Biochemistry Experiments. Higher Education Press, China,119-261.

Liu DL, Zhang X, Zhao YM, Wang NL, Yao XS (2016) Three new triterpenoid saponins from the roots of Ardisia crenata and their cytotoxic activities. Nat Prod Res 30, 2694-2703. https://doi.org/10.1080/14786419.2016.1146889

Ma L, Li W, Wang HG, Kuang XZ, Li Q, Wang YH, Xie P, Koike K (2015) A simple and rapid method to identify and quantitatively analyze triterpenoid saponins in Ardisia crenata using ultrafast liquid chromatography coupled with electrospray ionization quadrupole mass spectrometry. J Pharmaceut Biomed 102, 400-408. https://doi.org/10.1016/j.jpba.2014.10.013

McCallum JA, Walker JRL (1990) Phenolic biosynthesis during grain development in wheat: changes in phenylalanine ammonialyase activity and soluble phenolic content. J Cereal Sci 11, 35-49. https://doi.org/10.1016/S0733-5210(09)80179-3

McKinley DC, Rice CW, Blair JM (2008) Conversion of grassland to coniferous woodland has limited effects on soil nitrogen cycle processes. Soil Biol Biochem 40, 2627-2633. https://doi.org/10.1016/j.soilbio.2008.07.005

Mittler R (2002) Oxidative stress, antioxidants and stress tolerance. Trends Plant Sci 7, 405-410.

Moghimian N, Hosseini SM, Kooch Y, Darki B Z (2017) Impacts of changes in land use/cover on soil microbial and enzyme activities. Catena 157, 407-414. http://doi.org/10.1016/j.catena.2017.06.003 
Nottingham AT, Turner BL, Whitaker J, Ostle NJ, McNamara NP, Bardgett RD, Salina N, Meir P (2015) Soil microbial nutrient constraints along a tropical forest elevation gradient: a belowground test of a biogeochemical paradigm. Biogeosciences 12, $6071-6083$.

http://doi.org/10.5194/bg-12-6071-2015

Parras-Alcántara L, Lozano-García B, Galán-Espejo A (2015) Soil organic carbon along an altitudinal gradient in the Despeñaperros Natural Park, southern Spain. Solid Earth 6,125-134. https://doi.org/10.5194/se-6-125-2015

Podolak I, Mynarski A, Wróbel D, Grabowska K, Galanty A (2019) Bioactive benzoquinones content variability in red-berry and white-berry varieties of Ardisia crenata Sims and assessment of cytotoxic activity. Nat Prod Res https://doi.org/10.1080/14786419.2019.1614575

Purahong W, Durka W, Fischer M, Dommert S, Schöps R, Buscot F, Wubet T (2016) Tree species, tree genotypes and tree genotypic diversity levels affect microbe-mediated soil ecosystem functions in a subtropical forest. Sci Rep 6, 36672. https://doi.org/10.1038/srep36672

Raiesi F, Beheshti A (2014) Soil specific enzyme activity shows more clearly soil responses to paddy rice cultivation than absolute enzyme activity in primary forests of northwest Iran. Appl Soil Ecol 75, 63-70. http://dx.doi.org/10.1016/j.apsoil.2013.10.012

Raina H, Soni G, Jauhari N, Sharma N, Bharadvaja N (2014) Phytochemical importance of medicinal plants as potential sources of anticancer agents Turkish. J Bot 38, 1027-1035. https://doi.org/10.3906/bot-1405-93

Rajsnerová P, Klem K, Holub P, Novotná K, Večeřová K, Kozáčiková M, Rivas-Ubach A, Sardans J, Marek MV,

Peñuelas J, Urban $O$ (2014) Morphological, biochemical and physiological traits of upper and lower canopy leaves of European beech tend to converge with increasing altitude. Tree Physiol 35, 47-60. https://doi.org/10.1093/treephys/tpu104

Ren CJ, Kang D, Wu JP, Zhao FZ, Yang GH, Han XH, Feng YZ, Ren GX (2016) Temporal variation in soil enzyme activities after afforestation in the Loess Plateau, China. Geoderma 282, 103-111. https://doi.org/10.1016/j.geoderma.2016.07.018

Ruelland E, Vaultier MN, Zachowski A, Hurry V (2009) Cold Signalling and Cold Acclimation in Plants. Adv Bot Res 35-150. https://doi.org/10.1016/S0065-2296(08)00602-2

Saggar S, Mclntosh PD, Hedley CB, Knicker H (1999) Changes in soil microbial biomass, metabolic quotient, and organic matter turnover under Hieracium (H. pilosella L.). Biol Fertil Soils 30, 232-238.

Stone MM, DeForest JL, Plante AF (2014) Changes in extracellular enzyme activity and microbial community structure with soil depth at the Luquillo Critical Zone Observatory. Soil Biol Biochem 75, 237-247. https://doi.org/10.1016/j.soilbio.2014.04.017

Sundqvist MK, Wardle DA, Vincent A, Giesler R (2014) Contrasting nitrogen and phosphorus dynamics across an elevational gradient for subarctic tundra heath and meadow vegetation. Plant Soil 383, 387-399. https://doi.org/10.1007/s11104-014-2179-5

Terfa MT, Roro AG, Olsen J E, Torre S (2014) Effects of UV radiation on growth and postharvest characteristics of three pot rose cultivars grown at different altitudes. Sci Hortic-Amsterdam 178, 184-191. http://doi.org/10.1016/j.scienta.2014.08.021

Trasar-Cepeda C, Leiros MC, Gil-Sotres F (2008) Hydrolytic enzyme activities in agricultural and forest soils. Some implications for their use as indicators of soil quality. Soil Biol Biochem 40, 2146-2155. https://doi.org/10.1016/j.soilbio.2008.03.015

Ushio M, Kitayama K, Balser TC (2010) Tree species effects on soil enzyme activities through effects on soil physicochemical and microbial properties in a tropical montane forest on Mt.Kinabalu, Borneo. Pedobiologia 53, 227-233. https://doi.org/10.1016.j.pedobi.2009.12.003

Wang B Xue S, Liu GB, Zhang GH, Li G, Ren ZP (2012) Changes in soil nutrient and enzyme activities under different vegetations in the Loess Plateau area, Northwest China. Catena 92, 186-195. https://doi.org/10.1016/j.catena.2011.12.004

Wellburn AR, Lichtenthaler H (1984) Formulae and program to determine total carotenoids and chlorophylls A and B of leaf extracts in different solvents. Springer Netherlands 9-12.

Xiao L, Li P, Shi P, Liu Y (2018) Soil nutrient stoichiometries and enzymatic activities along an elevational gradient in the dry-hot valley region of southwestern China. Arch Agron Soil Sci 65, 322-333. https://doi.org/10.1080/03650340.2018.1502882

Xu HW, Qu Q, Lu BB, Li P, Xue S, Liu GB (2020) Response of soil specific enzyme activity to vegetation restoration in the Loess hilly region of China. Catena https://doi.org/10.1016/j.catena.2020.104564

Page 14/17 
Yu MQ, Meng GT, Li LP, Li GX, Chai Y (2014) The nitrogen mineralization of five forest types soil in eastern slope of Gaoligongshan Mountains. Adv Mater Res 222-228. http://doi.org/10.4028/www.scientific.net/AMR.1073-1076.222

Zhang XY, Dong WY, Dai XQ, Schaeffer S, Yang FT, Radosevich M, Xu LL, Liu XY, Sun XM (2015) Responses of absolute and specific soil enzyme activities to long term additions of organic and mineral fertilizer. Sci Total Environ 536, 59-67.

http://dx.doi.org/10.1016/j.scitotenv.2015.07.043

Zinn YL, Andrade AB, Araujo MA, Lal R (2018) Soil organic carbon retention more affected by altitude than texture in a forested mountain range in Brazil, Soil Res 56, 284-295. https://doi.org/10.1071/SR17205

\section{Figures}

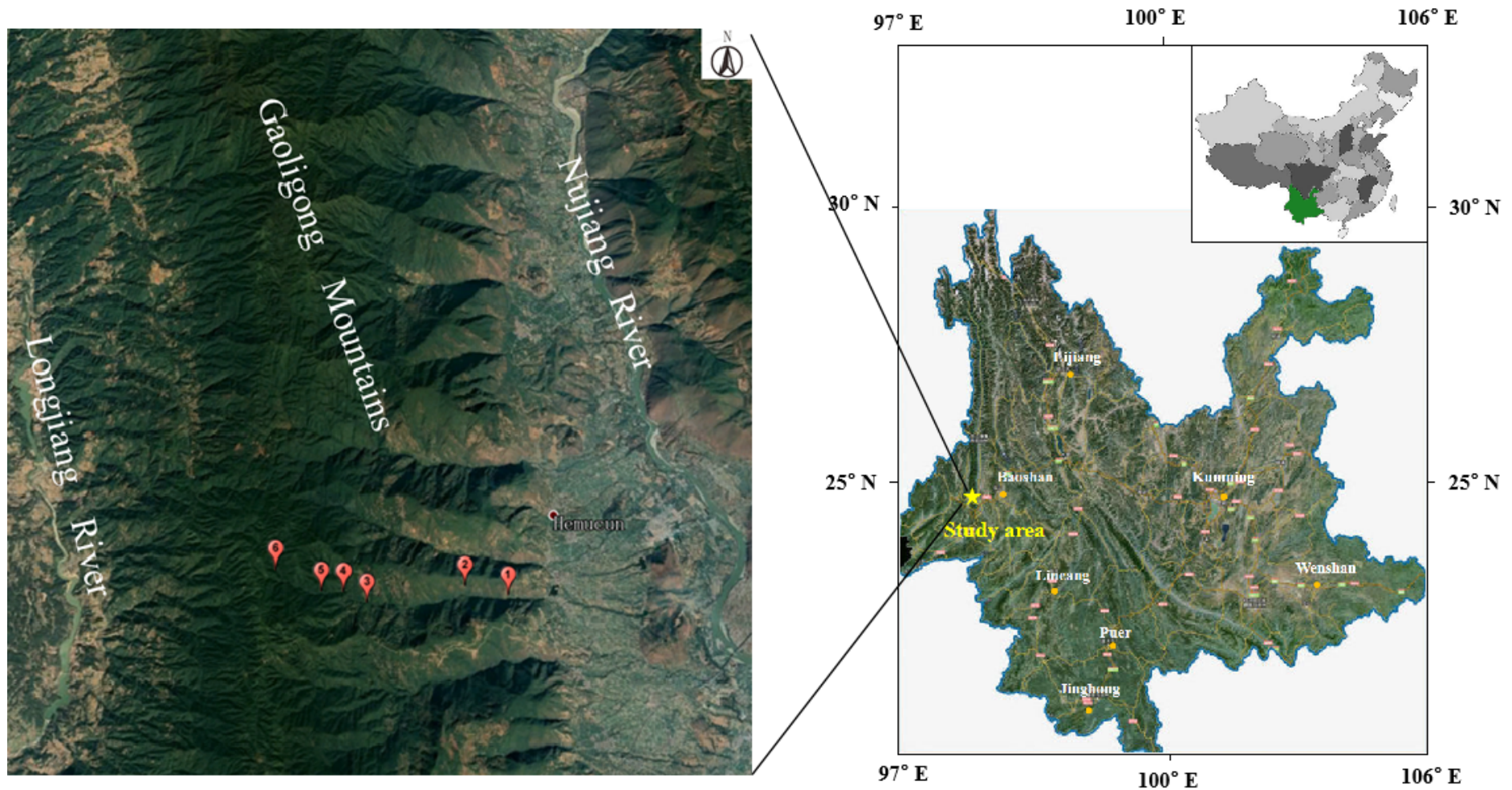

\section{Figure 1}

Location of study area Note: The designations employed and the presentation of the material on this map do not imply the expression of any opinion whatsoever on the part of Research Square concerning the legal status of any country, territory, city or area or of its authorities, or concerning the delimitation of its frontiers or boundaries. This map has been provided by the authors. 

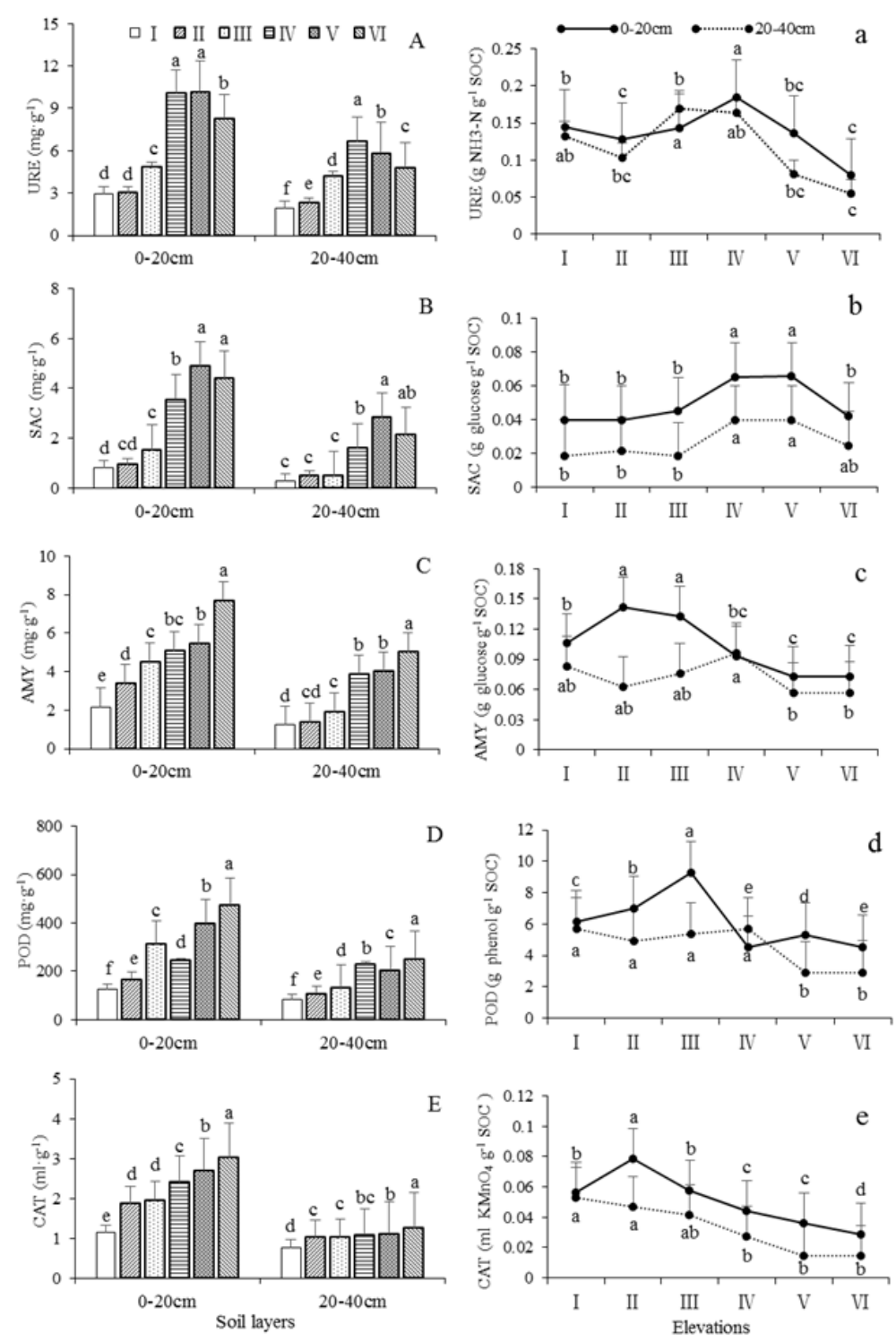

Figure 2

Soil absolute activities and specific enzyme activities per unit of SOC at different elevations and soil layers in the Gaoligong Mountain. Different letters indicate significant differences along the elevation gradients and layers $(P<0.05)$. 

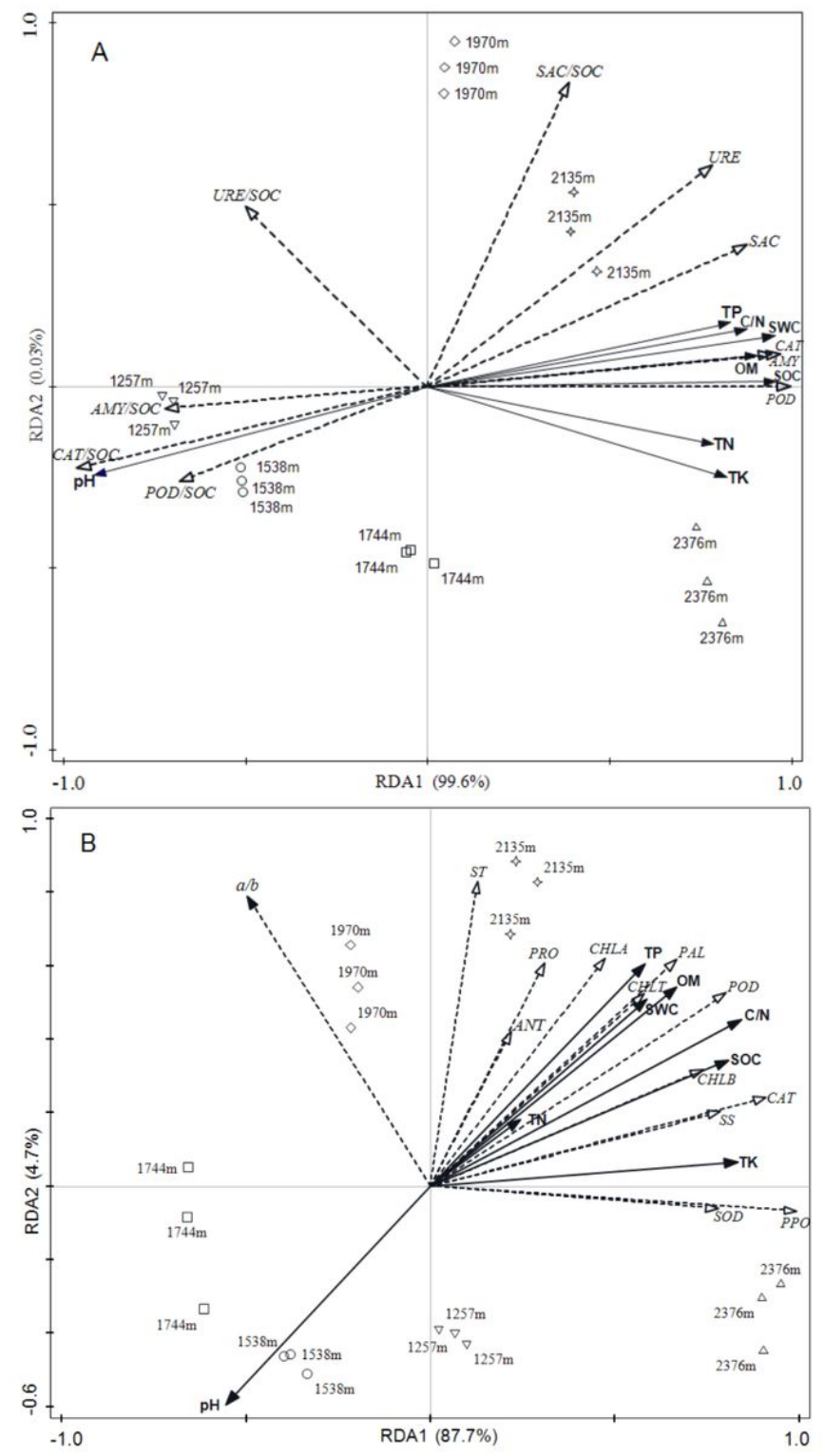

\section{Figure 3}

Redundancy analyses of the soil enzymatic activities and activities per unit of SOC (A), plant physiological characteristics (B) and environmental factors of A. crenata Sims var. bicolor

\section{Supplementary Files}

This is a list of supplementary files associated with this preprint. Click to download.

- SupplementaryTable.doc 